\title{
Development and Investigation of the Program Model of Multichannel Queueing System with Unreliable Recoverable Servers in Matlab Environment
}

\author{
Ramaz R. Shamugia ${ }^{1,2}$ \\ ${ }^{1}$ Research Department of Radiophysics and Electronic Systems Modeling, Ilia Vekua Sokhumi Institute of Physics and \\ Technologies, Tbilisi, Georgia \\ ${ }^{2}$ Faculty of Mathematics and Computer Science, Sokhumi State University, Tbilisi, Georgia \\ Email:rmz.shamugia@gmail.com
}

How to cite this paper: Shamugia, R.R. (2018) Development and Investigation of the Program Model of Multichannel Queueing System with Unreliable Recoverable Servers in Matlab Environment. Int. J. Communications, Network and System Sciences, 11, 229-237.

https://doi.org/10.4236/ijcns.2018.1111014

Received: October 1, 2018

Accepted: November 9, 2018

Published: November 12, 2018

Copyright $\odot 2018$ by author and Scientific Research Publishing Inc.

This work is licensed under the Creative

Commons Attribution International

License (CC BY 4.0).

http://creativecommons.org/licenses/by/4.0/

\section{(c) (i) Open Access}

\begin{abstract}
In real life, in different industries, we often deal with systems designed for multiple use for performing single-type tasks. Processes taking place at this time are called service of requirements, and the systems themselves-Queueing Systems. This article is dedicated to computer software modelling of processes taking place in the systems in question, Markov processes in particular. In this article, by means of Matlab environment, software realization of one of the typical models of queueing service theory-multichannel QS with unreliable recoverable servers and limited number of requirements in the system, is fulfilled. The results of this research are important because it gives the possibility to use received results to determine optimality degree of some real queueing systems that possess Markov property.
\end{abstract}

\section{Keywords}

Queueing System, Simulation, Queue, Modeling, Imitation, Complex System

\section{Introduction}

Modern computer and telecommunication systems and networks are complicated complexes of technical means that provide receiving, processing and transmitting of different data with definite quality parameters. They are widely represented in all fields of human life and activities, science and technology. The basis of such systems are computer systems of data receiving, processing and 
transmission with the use of electric, fiber-optic and radio-channels which as a matter of fact are Multichannel Queueing Systems (QS). At present, the field of above mentioned systems and networks are being considerably transformed due to development of wireless data transfer, data storage and processing (cloud computing), fiber optic technology etc. For further development of modern computer and telecommunication systems, it is needed to create appropriate mathematical and software models for their functioning that at the same time would take into consideration peculiarities appropriate to these systems [1] [2] [3].

It is known that computer operation and telecommunication systems can be greatly influenced by random factors that affect characteristics of their computer capacity, quality of data transfer etc. Examples of random factors could be artificial and natural noises, different levels of noise in the transmission channel, distance change between mobile transmitters, parallel transmission of high priority information, hardware failure and equipment breakdown, weather effects. Random factors especially have great influence over wireless network that is being developed intensively these days. Keeping in mind influence of random factors on the operation process is of great significance while building the adequate mathematical and simulation models and estimating performance calculations of telecommunication systems [4] [5] [6].

Mathematical methods of queueing system theory that function in a random environment allow to create adequate stochastic models of modern computer and telecommunication systems that take into consideration influence of random factors. Random environment or random medium means random process with final state of space independent from the system. In the case of fixed state of random medium the system functions as classical queueing system. But system parameters (incoming flow, service time distribution etc.) immediately change with the change of random medium state.

In this article multichannel queueing system is discussed which is according to Kendall's classification belongs to the type $A / B / m / K / M$, where $A$ is law of arrivals into the system, B-law of Service of Requirements coming into the service, $\mathrm{m}$-number of parallel-functioning equipment (service channel), $\mathrm{K}$ is admissible number of requirements in the system, i.e. total number of requirements waiting in a queue and those being served, and $M$ is the number of loading source. Whereas it is believed that system parameters are constant, but processes in the system are considered from the point of view of reproduction and death theory. The subject matter of the investigation in this article is scalable programming model of system with the simplest Poisson requirements arrivals and Exponential service flow [7] [8] [9].

The software model is developed on the basis of Kolmogorov's systems of differential equations with relation to probabilities of system states using similarly-named mnemonic rule. Keeping in mind solving equations of the mentioned system in the given starting conditions aspire to their established values that are called steady-state values of probability states, operation characteristics of the 
system are estimated on their basis.

\section{Statement}

In this research, while investigating computer and telecommunication management system presented in the form of queueing system consists of servers $m=$ $m 1+m 2$, where $m 1$ is number of main servers, and $m 2$ is number of standby servers operating in background, partial load mode.

Servers are being affected by refusals distributed according to Poisson law with total failure intensity $O=O 1+O 2$ where $O 1$ is failure intensity of main servers, and $O 2$ is failure intensity of standby servers. It is believed that servers operating in background mode are less affected by failures $(\mathrm{O} 1>\mathrm{O} 2)$.

In the system, the possibility of recovering of servers after failures is presupposed and it is fulfilled according to the demonstrative law with total intensity $V=V 1+V 2$, where $V 1$ is recovery intensity of main servers, and $V 2$ is recovery intensity of standby servers. It is supposed that standby servers would switch over to main mode operation for substituting main servers in case of failures, and servers returning to the system after recovery are also included.

In the above described system, Poisson arrival flow of requirements comes for being served with total intensity $M=M 1+M 2$ where $M 1$ is arrival intensity for main servers, and $M 2$ is arrival intensity for standby servers working in the background mode.

Serving arrival flow of requirements follows Exponential probability distribution law with total intensity $L=L 1+L 2$, where $L 1$ is service intensity by main servers, and $L 2$ is service intensity by standby servers.

It should be taken into consideration that in the system it is presupposed to have queue for arriving requirements coming the moment when all servers are busy serving requirements that have come earlier. Total number of arrivals that have possibility to be simultaneously in the system is limited, and equals to $K=$ $K 1+K 2+K 0$, where $K 1$ is the number of arrivals being served by main servers, $K 2$-is the number of arrivals being in the standby servers, and $K 0$ is the number of arrivals that are in the queue.

For characterizing system states $P_{i}(t)$-probabilistic functions are introduced that characterize transition from one state into another under the influence of flows taking place in the system (requirements arrival flow or queue discipline, failure flow and recoveries etc.) and defined as result of multiplication of probabilities of $\mathrm{i}$-state from which transition to appropriate intensity takes place. A key idea of Mnemonic rule of setting up Kolmogorov's equations is that derivative of probability of any state equals to total sum of probability flows that transit the system into this state, minus the sum of all probability flows that take out the system from this state. General view equation systems, formed according to this rule, looks like this:

$$
\frac{\mathrm{d} P_{i}(t)}{\mathrm{d} t}=-P_{i}(t) \sum_{\substack{j=1 \\ j \neq i}}^{K} \lambda_{i j}+\sum_{\substack{j=1 \\ j \neq i}}^{K} P_{j}(t) \lambda_{j i}, \text { where } \lambda_{i j}-\lambda_{j i}=0, i=1, \cdots, K ;
$$


where $P_{i}(t)$-transition probabilities between states of system, and $\lambda_{i j}$ and $\lambda_{j i}$-transition intensities between indicated states.

Having applied mnemonic rule to the above described system, in consequence of probabilistic reasoning, the system of differential equations can be written as (2) which determines transition probabilities between its states in the following form (it is widely known in literature):

$$
\frac{\mathrm{d} P_{i}(t)}{\mathrm{d} t}=-a_{i} P_{i}(t)+b_{i} P_{i-1}(t)+c_{i} P_{i+1}(t)
$$

where $P_{i}(t)=P_{i}(m 1, m 2 ; K 1, K 2, K 0 ; L 1, L 2 ; M 1, M 2 ; O 1, O 2 ; V 1, V 2 ; t)$ is probability of event whereby at the moment of time $t$ in the system, in case of given fixed parameter values $m 1, m 2 ; K 1, K 2, K 0 ; L 1, L 2 ; M 1, M 2 ; O 1, O 2 ; V 1, V 2$; there are i-requirements, but ratios in case of unknown functions are three-diagonal matrix with values estimated according the following formulae:

$$
\begin{aligned}
& a_{i}=i * L *(1-(M-O-V)) ; \\
& b_{i}=-\left(a_{i}+(-1) *(1-(M-O-V))\right) ; \\
& c_{i}=i * M *(1-(M-O-V)) ;
\end{aligned}
$$

To solve this system of equation with the help of Metlab medium, function ODE23 is used, presupposed for numeral integration of homogenous differential equation systems (HDE). It is applicable for solving simple differential equations, as well as for modelling complex dynamic systems. As it is known, any system of nonlinear HDE can be presented as system of differential equations of the first order in the explicit form of Cauchy $\mathrm{d} x / \mathrm{d} t=f(x, t)$, where $\mathrm{x}$ is vector of state, $t$ is time, $f$-nonlinear vector-function from disposal variables $x, t$.

Function $[t, X]=$ ode23 ("<function name $>$ ", t0, tf, $x 0$, tol, trace) integrates system HDE using Runge-Kutta's method of the second and forth order which have parameters: entry < function name>, that is the name of M-file, in which right parts of system HDE are computed, t 0 - is the initial value of time, tfinalis final value of time; $\mathrm{x} 0$-vector of initial conditions, tol-given precision (default to ode23, tol $=1 . \mathrm{e}-3$ ); trace-flag regulating exit middleware results (default to zero that suppresses exit of middleware results); output parameters: $t-$ current time; $\mathrm{X}$-two-dimensional arrays where every column corresponds to one variable.

\section{Development of Programming Model}

The programming model is built in the form of corresponding functions MMmK. The program presupposes computing ratios of three-diagonal matrix for the differential equation system-A, using appropriate functions Matlab: $\mathrm{A}=$ full (gallery('tridiag', n, a, b, c)), where a, b, c represent diagonal elements of matrix A.

\section{function MMmK;}

clc, close 


\section{$\%$ Initial system parameters}

DialogM = inputdlg ( $\{$ 'Number of main servers in the system (m1)'

'Number of secondary servers in the system (m2)'

'Number of seats in the queue for requirements (K0)'

'The maximum number of requirements that are on maintenance on the main servers (K1)'

'The maximum number of requirements that are on maintenance on secondary servers (K2)'

'Intensity of exponential maintenance on the main servers (L1)'

'Intensity of exponential maintenance on secondary servers (L2)'

'The intensity of the Poisson input stream of requirements on the main servers (M1)'

'The intensity of the Poisson input stream of requirements on the secondary servers (M2)

'The intensity of the Poisson failure flow of the main servers (O1)' 'The intensity of the Poisson failure flow of the secondary servers (O2)'

'The intensity of the exponential recovery flow of the main servers (V1)'

'The intensity of the exponential recovery flow of the secondary servers (V2)'\}),

'Enter the initial system parameters';

$\mathrm{m} 1=$ str2double (Dialog $\mathrm{M}\{1\}$ ); \% Number of main servers in the system $\mathrm{m} 2=$ str2double (Dialog $\mathrm{M}\{2\}$ ); \% Number of secondary servers in the system $\mathrm{K} 0=$ str2double (Dialog $\mathrm{M}\{3\}$ ); \% Number of seats in the queue for requirements

$\mathrm{K} 1=$ str2double (Dialog $\mathrm{M}\{4\}$ ); \% The maximum number of requirements that are on maintenance on the main servers

$\mathrm{K} 2=$ str2double (Dialog $\mathrm{M}\{5\}$ ); \% The maximum number of requirements that are on maintenance on secondary servers

$\mathrm{L} 1=$ str2double (Dialog $\mathrm{M}\{6\}$ ); \%intensity of exponential maintenance on the main servers

L2=str2double (Dialog $\mathrm{M}\{7\})$; \%intensity of exponential maintenance on secondary servers

M1=str2double (Dialog M $\{8\}$ ); \%The intensity of the Poisson input stream of requirements on the main servers

M2=str2double (DialogM $\{9\})$; $\quad$ \%The intensity of the Poisson input stream of requirements on the secondary servers

$\mathrm{O} 1=$ str2double (Dialog $\mathrm{M}\{10\})$; $\quad$ The intensity of the Poisson failure flow of the main servers

O2=str2double (Dialog $\mathrm{M}\{11\})$; \%The intensity of the Poisson failure flow of the secondary servers

V1=str2double (Dialog $\mathrm{M}\{12\})$; \%The intensity of the exponential recovery flow of the main servers 
V2=str2double (Dialog $\mathrm{M}\{13\}$ ); \%The intensity of the exponential recovery flow of the secondary servers

\section{global A}

syms n k a b c L N O V K M m m1 m2 M M1 M2 L L1 L2 O O1 O2 V V1 V2 K K0 K1 K2 t T P

$\mathrm{L}=\mathrm{L} 1+\mathrm{L} 2 \quad \%$ The total intensity of exponential flow of service of requirements on the servers of the system

$\mathrm{M}=\mathrm{M} 1+\mathrm{M} 2 \%$ The total intensity of the Poisson flow of incoming requirements

$\mathrm{O}=\mathrm{O} 1+\mathrm{O} 2 \quad \%$ The total intensity of the Poisson flow of failures of servers $\mathrm{V}=\mathrm{V} 1+\mathrm{V} 2 \quad \%$ The total intensity of the exponential flow of recovery of servers $\mathrm{K}=\mathrm{K} 1+\mathrm{K} 2+\mathrm{K} 0$;

for $\mathrm{i}=1: \mathrm{K}$

$$
\begin{aligned}
& a=i^{\star} L ; \\
& b=-\left(a+(i-1)^{\star}(M-O-V)\right) ; \\
& c=i^{\star} M ; \\
& A=\text { full(gallery ('tridiag',K,a,b,c,'double')); }
\end{aligned}
$$

\section{$\% \%$ Numerical solution of differential equations}

$$
\begin{aligned}
& \mathrm{P} 0=[1 ; \mathrm{zeros}(\text { length }(\mathrm{A})-1,1)] \\
& \mathrm{T}=[0,0.01] ; \\
& \text { [t,P] = ode23(@ cmo, T, P0); } \\
& \quad \text { End }
\end{aligned}
$$

\section{$\% \%$ Constructing of diagrams of states probabilities}

line $\left(\mathrm{t}, \mathrm{P}, \mathrm{llinew}^{\prime}, 2\right)$

line (t,P(:,K),'linew',2, 'color','r') \% \% P(K-1)

grid on

$\mathrm{N}$ = length(A)-1;

arr $=[0: \mathrm{N}]^{\prime}$;

$\operatorname{str}=$ num2str(arr);

legend (strcat('|bflitP\rm|bf_', str, '(|itt|rm|bf)'));

title(sprintf('\%s Probabilitys of system states M/M/\%d/\%d', 'Ibfffontsize\{12\}',i, $\mathrm{K})$ );

xlabel('|bflit|fontsize\{12\} Model Time ')

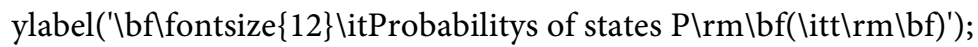

set(gca, 'fontweight','bold', 'fontsize',10)

fprintf('In Stationary probabilities: In');

for $\mathrm{J}=1:$ length $(\mathrm{A})$

fprintf ('\tP\%d = \%丹ln', J-1, P(end,J));

end 
fprintf ('In\t Operational characteristics: $\mid n ')$;

Pnot $=\mathrm{P}($ end, end $)$;

fprintf ('Probabilities of failures Pnot $=\% \mathrm{f} \backslash \mathrm{n}$ ', $\mathrm{P}(\mathrm{end}$,end));

$\mathrm{Q}=1$ - Pnot;

Fprintf ('Relative throughput $\mathrm{Q}=\% \mathrm{f} \mathrm{n}$ ', $\mathrm{Q}$ );

$\mathrm{Ab}=\mathrm{L}^{\star} \mathrm{Q}$;

fprintf ('Absolute throughput $\mathrm{Ab}=\% \mathrm{f} \cap \mathrm{n}$ ', $\mathrm{Ab}$ );

$\mathrm{Pq}=\operatorname{sum}(\mathrm{P}($ end, $\mathrm{i}+1$ :end $))$;

Fprintf ('Queue length probability $\mathrm{Pq}=\% \mathrm{fn}$ ', Pq);

Ps $=\operatorname{sum}(\mathrm{P}(\mathrm{end}, \mathrm{i}$ :end $)$;

Fprintf (' The probability of all servers being busy Ps = \%fn', Ps);

Ns $=[0: \text { length }(\mathrm{A})-1]^{*} \mathrm{P}($ end,:)';

fprintf('The average number of requirements in the system Ns $=\% \mathrm{fn}$ ', Ns);

fprintf('Average time of location of requirements in the system Ts $=\% \mathrm{fln}$ ', $\mathrm{Ns} / \mathrm{L})$;

$\mathrm{Nq}=[0:(\mathrm{K}-\mathrm{i})]^{*} \mathrm{P}(\mathrm{end}, \mathrm{i}: \mathrm{K})^{\prime} ;$

fprintf('Average queue length $\mathrm{Nq}=\% \mathrm{fn}$ ', $\mathrm{Nq}$ );

fprintf('Average time of location of requirements in the queue $\mathrm{Tq}=\% \mathrm{f} / \mathrm{n}$ ', $\mathrm{Nq} / \mathrm{L})$;

function $\mathrm{f}=\mathrm{cmo}(\mathrm{t}, \mathrm{P})$;

$\% \%$ Functions describing the right-hand sides of differential equations

global A

$\mathrm{f}=\mathrm{A}^{\star} \mathrm{P}$

$\%$ Results of program execution

$\%$ Stationary probabilities

\%The diagram of states probabilities of the system
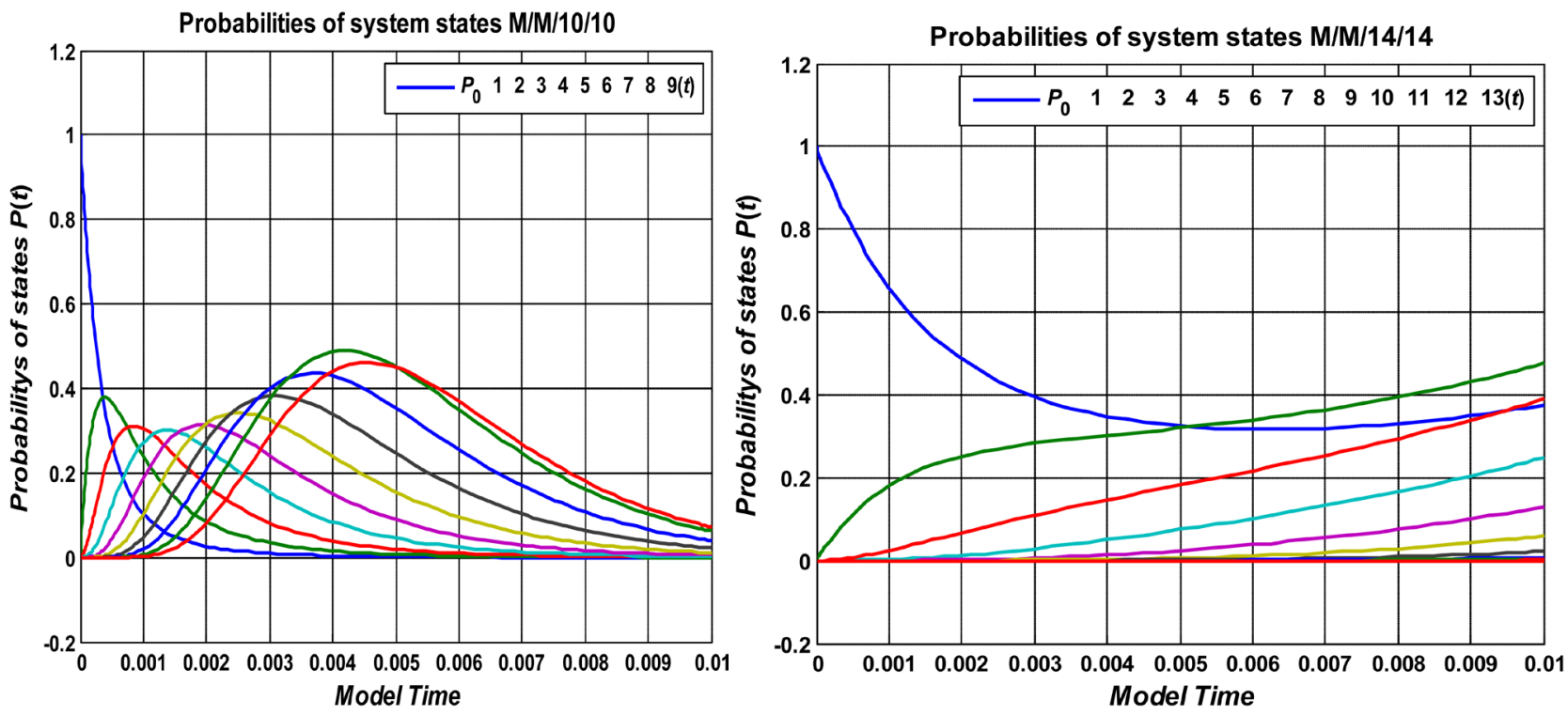

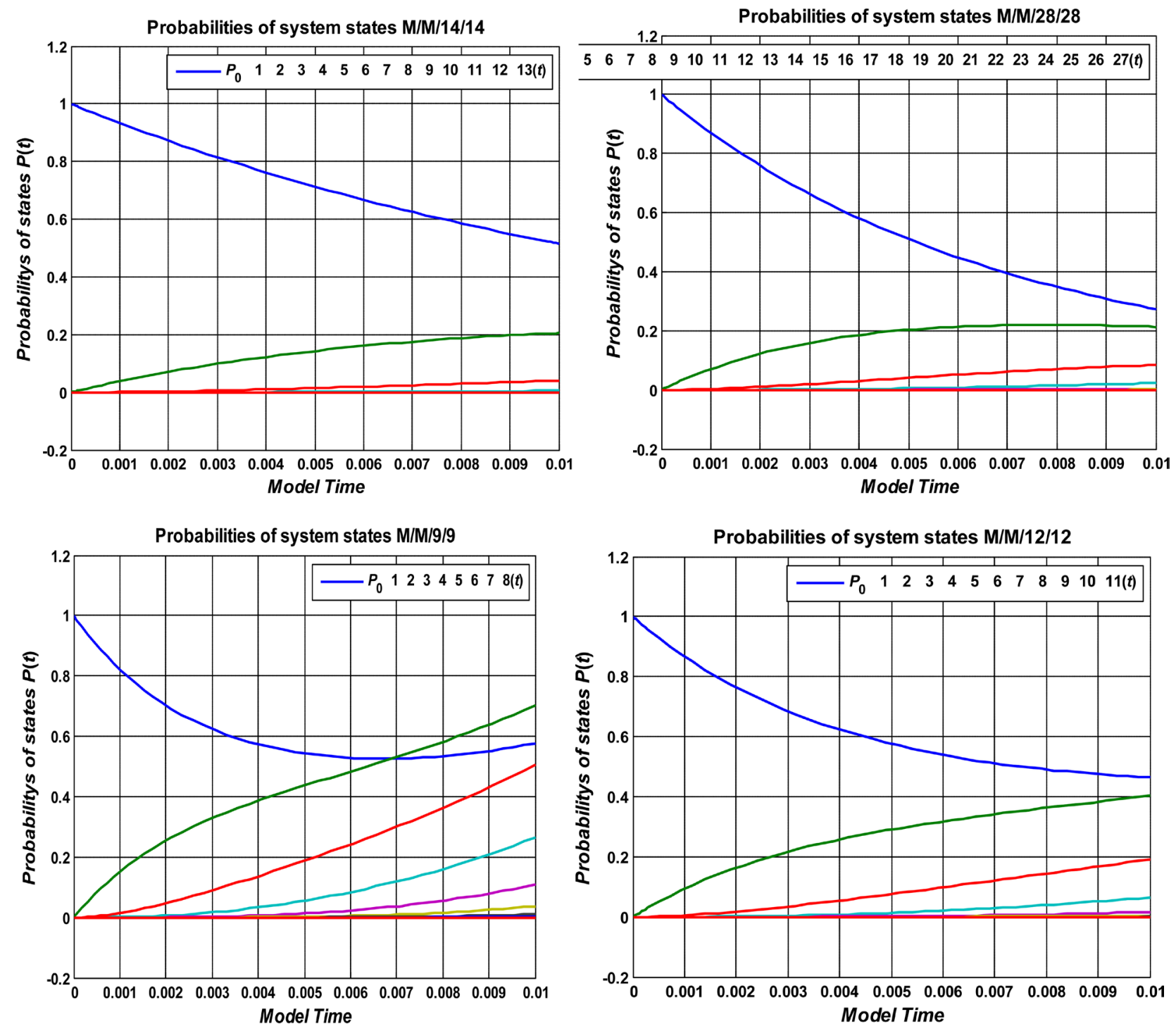

\section{Conclusion}

Bearing in mind the significance of computer modelling of complex computer and telecommunication systems presented in the form of QS, in the proposed work, on the basis of Markovian processes of birth and death, scalable program model of QS has been developed with limited number of requirements in the system that allows to determine some probabilistic characteristics of processes happening in them. Program model is created in the Matlab environment that allows graphic presentation of the results. Practical implementation of similar models is highly demanded. Such models significantly simplify researching of queueing systems, they give clear comprehension of states of considered systems, allow to optimize their work.

\section{Conflicts of Interest}

The author declares no conflicts of interest regarding the publication of this paper. 


\section{References}

[1] Saati, T.L. (1965) Elements of Queuing Theory and Its Application. Sovetskoe Radio, 510 .

[2] Cherkesov, G.N. (1974) Dependability of Technical Systems with Time Redundancy. Sovetskoe Radio, 296.

[3] Gnedenko, B.V. and Kovalenko, I.N. (2012) Introduction to Queuing Theory. LKT, 400.

[4] Feller, W. (1971) An Introduction to Probability Theory and Its Applications. Vol. 2, John Willey and Sons, New York, 766.

[5] [Khurodze, R.A., Shamugia, R.R. and Kukava, R.R. (2006) Research of Dependability Characteristics of Technical Systems with Equipment and Time Duplications. Bulletin of the Georgian National Academy of Sciences, 174, 110-113.

[6] Shamugia, R.R. (2014) On One Model of Complex Technical Queuing System with Unreliable Devices and with Time Redundancy. International Journal of Communications, Network and System Sciences, 7, 257-264.

http://file.scirp.org/Html/1-9701840_48353.htm

[7] Shamugia, R.R. (2014) On One Model of Multichannel Queuing System with Unreliable Repairable Servers and Input Memory. International Journal of Communications, Network and System Sciences, 7, 279-285.

http://www.scirp.org/journal/PaperInformation.aspx?PaperID=48474

[8] Shamugia, R.R. (2015) On One Analytical Model of a Probability Estimation of Quality and Efficiency of Functioning of Complex Technical Queuing Systems. International Journal of Communications, Network and System Sciences, 8, 295-303. http://www.scirp.org/journal/PaperInformation.aspx?PaperID=58537

[9] Shamugia, R.R. (2016) Probabilistic Model of Technical Queuing Systems with Subsystems for Detection and Recovery of Failures. International Journal of Communications, Network and System Sciences, 9, 305-310. http://www.scirp.org/journal/PaperInformation.aspx?PaperID=69525 